\title{
Progression of White Matter Hyperintensities Contributes to Lacunar Infarction
}

\author{
Xin Xu ${ }^{1,2,3, \# \text {, Yuanyuan Gao }}{ }^{1,2,3, \#}$, Renyuan Liu ${ }^{1,2,3}$, Lai Qian ${ }^{1,2,3}$, Yan Chen ${ }^{1,2,3}$, Xiaoying Wang ${ }^{4}$, \\ Yun $\mathrm{Xu}^{1,2,3^{*}}$
}

1Department of Neurology, Affiliated Drum Tower Hospital, and Jiangsu Key Laboratory for Molecular
Medicine, Nanjing University Medical School, Nanjing 210008, China.
${ }^{2}$ Jiangsu Province Stroke Center for Diagnosis and Therapy, Nanjing 210008 , China.
${ }^{3}$ Nanjing Neuropsychiatry Clinic Medical Center, Nanjing 210008, China.
${ }^{4}$ Departments of Neurology, Massachusetts General Hospital, Harvard Medical School, Charlestown, MA, USA.

[Received July 14, 2017; Revised August 4, 2017; Accepted August 8, 2017]

\begin{abstract}
Both white matter hyperintensities (WMHs) and lacunar infarctions (LIs) are magnetic resonance imaging (MRI) markers of cerebral small vessel disease (SVD). However, the association between WMH and LI remains unclear. In this study, we asked whether WMH progression is related to LI occurrence using retrospective data. Overall, $8475 \mathrm{WMH}$ patients with at least two MRI images were screened, and 187 patients were included in the final study; 76 patients had WMH with LI (WL), and 111 patients had WMH without LI (WOL). The 187 patients were divided into three groups according to WMH progression: Group 1 (no progression), Group $2(0-53.64 \% \mathrm{WMH}$ progression) and Group 3 ( $\geq 53.64 \% \mathrm{WMH}$ progression). We found that both WMH volumes and Fazekas scores were higher in WL patients compared with those in WOL patients according to the $1^{\text {st }}$ and $2^{\text {nd }}$ MRI images $(P<0.001)$, whereas WMH progression was not significantly different between these two groups $(P>0.05)$. Importantly, we found that the occurrence rates for $L I$ were increased in Groups 2 and 3 compared with those in Group 1. Multiple logistic regression analysis demonstrated that the risk of LI occurrence was significantly increased in Group 2 versus that in Group 1 (odds ratio, 3.36; 95\% CI, 1.48 to $7.67 ; \mathrm{P}=0.004)$ after adjusting for the baseline patient characteristics and the interval between the two MRI scans. Additionally, with a stratification time of less than 24 months, the risk of LI occurrence was higher in Group 2 versus that in Group 1, after adjusting for baseline confounding factors (odds ratio, 3.68; 95\% CI, 1.51 to 8.99; $P=0.004)$. In conclusion, we found that $\mathrm{WMH}$ progression was significantly associated with $\mathrm{LI}$ occurrence, particularly within the first two years, and that this progression could serve as an independent indicator of LI development.
\end{abstract}

Key words: progression of white matter hyperintensities, lacunar infarction, incidence, quantitative analysis

White matter hyperintensities (WMHs) and lacunar infarctions (LIs) are the primary MRI representations of cerebral small vessel disease (SVD). SVD accounts for up to $25 \%$ of stroke and $50 \%$ of dementia cases [1-4] and is characterized by stereotypical radiological changes in
MRI, including WMHs, LI, lacunes, enlarged perivascular space, cerebral microbleeds and brain atrophy [5]. SVD is also associated with vascular risk factors, particularly hypertension [6]. The clinical spectrum for SVD ranges from asymptomatic disease,

*Correspondence should be addressed to: Yun Xu MD, PhD, Department of Neurology, Affiliated Drum Tower Hospital of Nanjing University Medical School, Nanjing 210008, China. Email: xuyun20042001@aliyun.com

Copyright: ( $2017 \mathrm{Xu} \mathrm{X}$ et al. This is an open-access article distributed under the terms of the Creative Commons Attribution License, which permits unrestricted use, distribution, and reproduction in any medium, provided the original author and source are credited. 
which is detectable by brain imaging in healthy individuals, to extensive $\mathrm{WMH}$ and lacunar infarcts in vascular dementia [7, 8]. Approximately $70 \%$ of people over 65 years of age present with varying degrees of WMH after undergoing MRI $[9,10]$.

WMHs from SVD are thought to be caused by chronic ischaemia attributable to arteriolosclerosis of medullary arteries [11]. By contrast, LI is an acute ischaemic lesion of SVD primarily caused by segmental arterial disorganization and is usually secondary to hypertension [1]. Both WMH and LI are associated with age and hypertension [12, 13], and because these two pathologies share similar risk factors, they are often observed together. Large WMH volumes are clinically important because they are associated with cognitive impairment, dementia, gait disturbances and depression $[14,15]$. Although most LIs appear to be "silent," meaning they are not accompanied by stroke-like symptoms, they are thought to be associated with subtle cognitive dysfunction $[16,17]$. However, the relationship between WMH volume and LI remains unclear. In this study, we aimed to determine whether WMH progression is related to LI occurrence.

\section{MATERIALS AND METHODS}

\section{Study population}

A total of 8475 patients with WMHs on two or more MRI scans were studied retrospectively from January 1, 2011 to December 1, 2016 from the Drum Tower hospital imaging register centre of Nanjing University Medical School. Patients were recruited if they had WMHs on FLAIR images from their first MRI and had complete clinical data, including demographic data, a medical history, a physical examination and a laboratory examination. Exclusion criteria included cerebral infarction, cerebral haemorrhage, subarachnoid haemorrhage, intracranial and extracranial vascular lesions (Narrow degree over 50\%), cerebral venous sinus thrombosis, central nervous system infections, leukoencephalopathy of nonvascular origin (immunological, demyelinating, metabolic, toxic, infection or other) or severe unrelated neurological diseases (e.g., cardiac, hepatic, or renal failure, cancer or other relevant systemic diseases). Consequently, 8288 patients were ruled out with 1264 patients lacking complete clinical data, 641 with ischaemic stroke, 70 with cerebral haemorrhage, 19 with intracranial infection, 8 with intracranial venous sinus thrombosis, 2674 with tumours, 191 patients lacking FLAIR images, and 3421 patients for other reasons. In the end, 187 patients were analysed in this study, including 76 patients with LI (WL) and 111 without LI (WOL) (Fig. 1). The Affiliated Drum Tower Hospital of Nanjing University Medical School Ethics Committee approved this study. The procedures were conducted according to institutional guidelines.

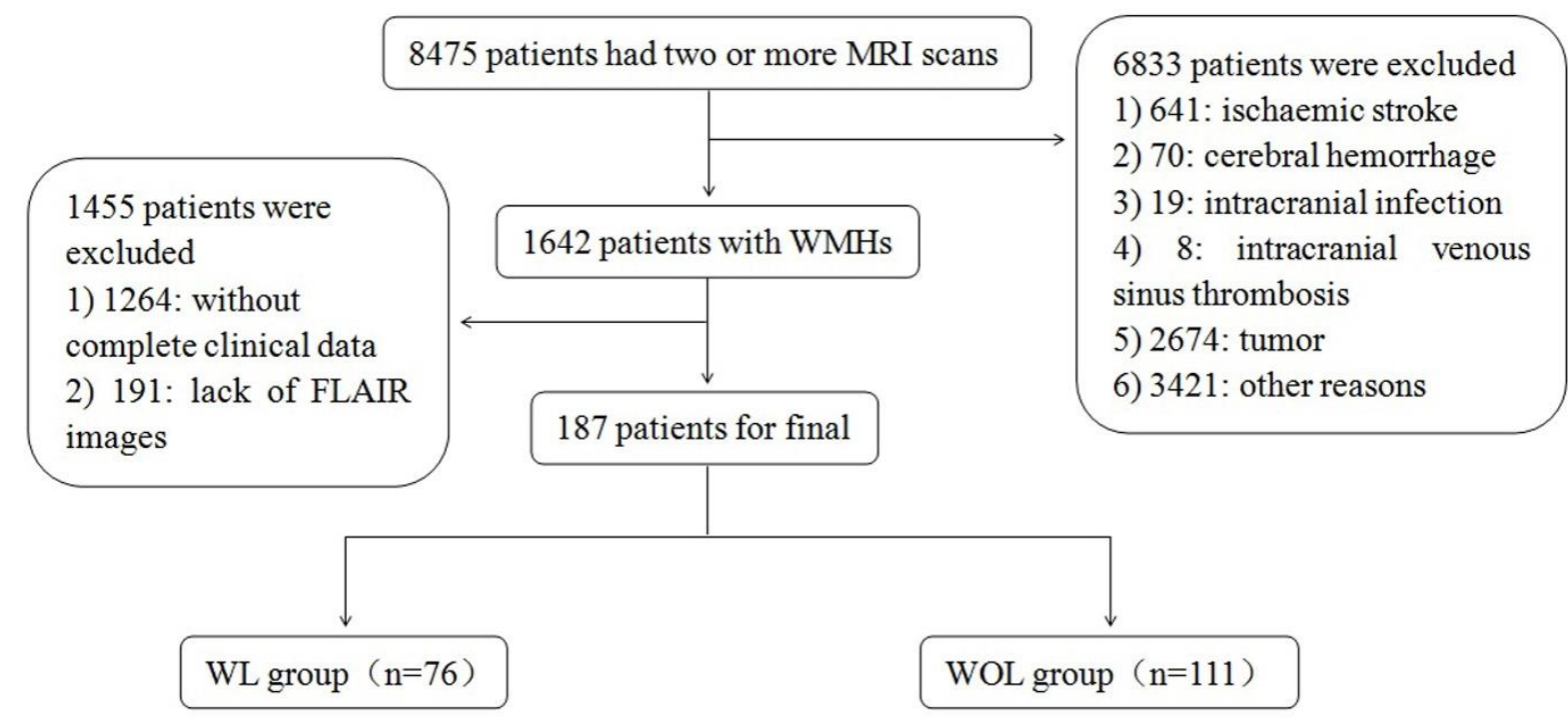

Figure 1. Diagrammatic sketch of the screening process. 

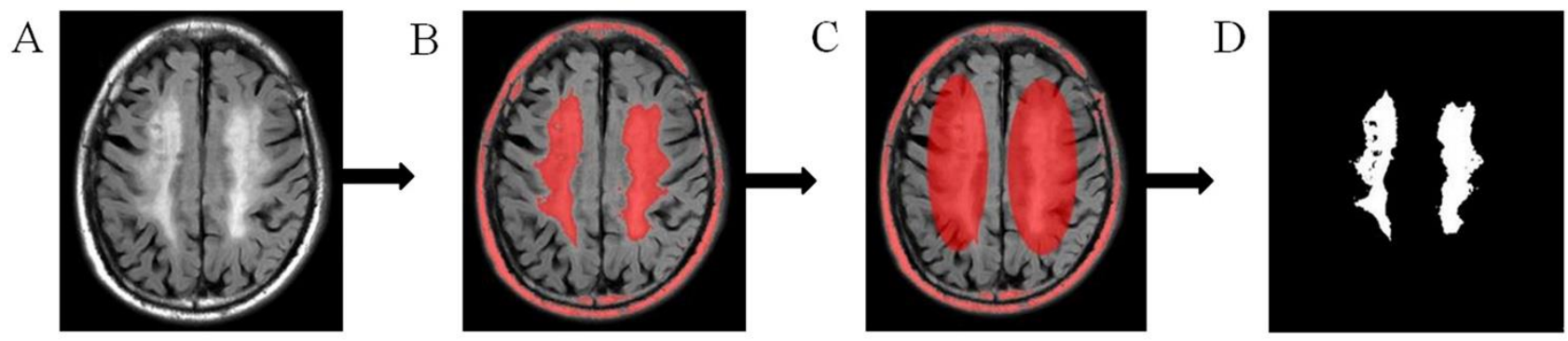

Figure 2. Quantitative steps of WMH volume. (A) Original FLAIR image. (B) The high signal area. (C) A sketch of the effective $\mathrm{WMH}$ area. (D) Extract of the effective WMH area.

\section{Risk factors}

To assess relevant risk factors at baseline, we determined patient demographics (age, sex and medical history), history of hypertension, history of diabetes mellitus, clinical history of stroke, atrial fibrillation and myocardial infarction, history of dyslipidaemia, and past or present cigarette or alcohol use. Patients underwent a physical examination, systolic and diastolic blood pressure were measured, and laboratory examinations were performed, including tests for glucose (random blood glucose, fasting blood glucose, postprandial blood glucose and glycosylated haemoglobin), total cholesterol, highdensity lipoprotein, triglycerides, low-density lipoprotein, apolipoprotein A-I, urea nitrogen, creatinine, uric acid and $\mathrm{C}$-reactive protein.

\section{MRI examinations}

MRI scanning was performed on a $1.5 \mathrm{~T}$ or $3.0 \mathrm{~T}$ scanner. The scanning protocol included a whole brain T1 magnetization-prepared rapid gradient echo sequence, a transversal T2-weighted turbo spin echo sequence, and a fluid-attenuated inversion recovery (FLAIR) pulse sequences.

\section{Radiological diagnosis of WMHs and LI}

WMHs are hyperintense on T2-weighted or FLAIR sequences, and they can appear as isointense or hypointense (although they are not as hypointense as CSF) on T1-weighted sequences, depending on the sequence parameters and severity of the pathological changes [5]. White matter lesions, characterized by bilateral and mostly symmetrical hyperintensities on T2-weighted MRI, are common in older individuals.

LI is a small subcortical infarct with a diameter ranging from 3-15 $\mathrm{mm}$ on axial sections [5].

\section{Quantitative analysis of WMH volume}

WMH volumes were quantified using the software programmes MRICRON (University of Nottingham School of Psychology, Nottingham, UK; www.mricro.com) and ITK-SNAP (University of Pennsylvania, Philadelphia, USA; www.itksnap.org) (Fig. 2). All scans were checked by visual inspection. First, we used the MRICRON software to extract the effective WMH area, and then we used the ITK-SNAP software to calculate the volume. To eliminate individual differences, WMH volume was standardized by intracranial volume (Standardized $\mathrm{WMH}$ volume $=$ actual calculated $\mathrm{WMH}$ volume/intracranial volume $\times 100 \%$ ). As baseline $\mathrm{WMH}$ volume is a strong predictor of WMH progression, we calculated WMH progression as a percentage of the baseline WMH volume using the formula WMH progression $=(\mathrm{WMH} 2-\mathrm{WMH} 1) / \mathrm{WMH} 1 * 100[15,18]$. Based on the state of WMH progression, patients were divided into three groups: no WMH progression group (Group 1) and a WMH progression group segmented into two subgroups (Group 2 and Group 3) according to the median measurement (non-normal distribution).

\section{Fazekas scale score}

The degree of WMH severity was rated visually on axial FLAIR images using the modified Fazekas scale [19, 20], which is the most widely used and validated system for describing WMH severity. The scale divides WMHs into periventricular and deep, and periventricular WMHs were graded according to the following patterns: $0=$ absent; 1 = caps or pencil-thin lining; $2=$ smooth halo; and $3=$ irregular periventricular WMH extending into a deep WMH. Deep WMHs were graded according to the following patterns: $0=$ absent; $1=$ punctate foci; $2=$ beginning confluence of foci; and 3 = large fused areas. A total score ( 0 to 6 ) was acquired by adding the periventricular and deep WMH scores, and the results 
were compared using quantitative analyses.

\section{Statistical analysis}

Data for the baseline characteristics were calculated separately for the two groups with and without lacunar infarction. Mean (standard deviation) or median (interquartile ranges) measurements were chosen depending on whether continuous variables had a normal distribution. Frequencies were used to describe categorical variables. Differences between groups were tested using an independent Student's t-test for normally distributed continuous variables, with a Wilcoxon rank- sum test for variables that followed a skewed distribution, and a chi-squared test for categorical variables. Univariate and multivariate logistic regression analyses were used to assess the association between WMH progression and LI. The ORs and $95 \%$ CIs of WMH progression were calculated with no WMH progression as a reference. Potential covariates, such as age, gender, hypertension, the interval between the two MRI scans and others, were adjusted in the multivariate model. All $\mathrm{P}$ values were twotailed, and $\mathrm{P}<0.05$ was considered statistically significant. Statistical analyses were performed using the SAS statistical package (version 9.3).

Table 1. Baseline characteristics.

\begin{tabular}{|c|c|c|c|}
\hline & WL $(n=76)$ & WOL $(n=111)$ & P Value \\
\hline Age, mean \pm SD & $72.45 \pm 10.93$ & $68.37 \pm 10.81$ & $0.013 *$ \\
\hline Men, n (\%) & $52(68.42)$ & $61(54.95)$ & 0.064 \\
\hline Smoking, n (\%) & $12(15.79)$ & $13(11.71)$ & 0.421 \\
\hline Alcohol, n (\%) & $4(5.26)$ & $7(6.31)$ & 0.766 \\
\hline $\mathrm{SBP}$, mean $\pm \mathrm{SD}$ & $139.33 \pm 19.19$ & $131.47 \pm 14.91$ & $0.002 * *$ \\
\hline $\mathrm{DBP}$, mean $\pm \mathrm{SD}$ & $76.36 \pm 12.44$ & $73.77 \pm 11.59$ & 0.147 \\
\hline FPG, median (IQR) & $5.27(4.69-6.74)$ & $5.21(4.60-6.39)$ & 0.391 \\
\hline RBG, median (IQR) & $7.05(5.60-9.00)$ & $7.20(6.00-8.90)$ & 0.577 \\
\hline PBG, median (IQR) & $10.40(7.65-12.85)$ & $8.00(7.00-11.00)$ & $0.005 * *$ \\
\hline HbA1c, median (IQR) & $6.00(5.60-6.70)$ & $6.00(5.50-6.70)$ & 0.993 \\
\hline TC, median (IQR) & $3.81(3.14-4.67)$ & $4.12(3.58-4.90)$ & $0.027 *$ \\
\hline TG, median (IQR) & $1.33(0.86-1.89)$ & $1.34(0.95-1.96)$ & 0.414 \\
\hline LDL, median (IQR) & $1.96(1.35-2.57)$ & $2.21(1.74-2.72)$ & 0.121 \\
\hline HDL, median (IQR) & $1.02(0.89-1.15)$ & $1.05(0.86-1.25)$ & 0.337 \\
\hline ApoA I, median (IQR) & $1.06(0.95-1.27)$ & $1.15(1.04-1.38)$ & $0.009 * *$ \\
\hline BUN, median (IQR) & $5.40(4.40-6.96)$ & $5.20(4.30-6.30)$ & 0.347 \\
\hline Cr, median (IQR) & $70.50(61.50-89.50)$ & $66.00(56.00-78.00)$ & $0.044 *$ \\
\hline UA, median (IQR) & $315.5(243.5-380)$ & $315(268-384)$ & 0.412 \\
\hline CRP, median (IQR) & $4.1(3.0-10.3)$ & $3.7(2.5-4.8)$ & $0.004 * *$ \\
\hline Hypertension, n (\%) & $37(48.68)$ & $34(30.63)$ & $0.013 *$ \\
\hline Diabetes mellitus, n (\%) & $44(57.89)$ & $48(43.24)$ & $0.049 *$ \\
\hline Dyslipidaemia, n (\%) & $46(60.53)$ & $60(54.05)$ & 0.380 \\
\hline Interval time, n (\%) & $19(11-29)$ & $15.5(10-27)$ & 0.2931 \\
\hline
\end{tabular}

$\overline{\mathrm{WMH}}=$ White matter hyperintensities; $\mathrm{LI}=$ Lacunar infarction; $\mathrm{WL}=\mathrm{WMH}$ with LI; WOL $=\mathrm{WMH}$ with no lacunar infarction; SBP $=$ Systolic blood pressure; $\mathrm{DBP}=$ Diastolic blood pressure; $\mathrm{FPG}=$ Fasting blood glucose; $\mathrm{RBG}=$ Random blood glucose; $\mathrm{PBG}=$ Postprandial blood glucose; $\mathrm{HbA} 1 \mathrm{c}=$ Glycosylated haemoglobin; TC = Total cholesterol; TG = Triglyceride; $\mathrm{LDL}=$ Low density lipoprotein; HDL = High density lipoprotein; ApoA I = Apolipoprotein A I; BUN = Blood urea nitrogen; $\mathrm{Cr}=$ Creatinine; $\mathrm{UA}=$ Uric acid; $\mathrm{CRP}=\mathrm{C}$-reactive protein

* Significant difference $(\mathrm{P}<0.05)$; ** Significant difference $(\mathrm{P}<0.01)$. 


\section{RESULTS}

\section{Baseline characteristics}

As shown in Table 1, multiple variables were different between the WL and WOL groups, including age, systolic blood pressure (SBP), postprandial blood glucose (PBG), total cholesterol (TC), apolipoprotein A-I (ApoA I), creatinine $(\mathrm{Cr})$, C-reactive protein (CRP), diabetes mellitus and hypertension. Most of those variables were higher in the WL group $(\mathrm{P}<0.05)$, although TC and ApoA I were reduced relative to the WOL groups $(\mathrm{P}<0.05)$.

WMH volume and Fazekas scale scores were different between WL and WOL groups

Based on MRI data from the $1^{\text {st }}$ and $2^{\text {nd }}$ scans, WMH

Table 2. Relationship between WMH Progression and LI. volumes were significantly higher in WL compared with WOL $(\mathrm{P}<0.001$, Fig. 3A). However, there were no significant differences in the progression of $\mathrm{WMH}$ volumes between WL and WOL ( $\mathrm{P}=0.185$, Fig. 3B). Similarly, Fazekas scale scores of WMH showed the same statistical trends (Fig. 3C and D).

\section{LI occurred more frequently in patients with $\mathrm{WMH}$ progression}

Next, we studied the distributions of LI occurrence in groups with different WMH progression. As shown in Figure $4,25 \%$ of patients had LI in Group 1, whereas $52.3 \%$ and $42.4 \%$ of patients had LI in Groups 2 and 3, respectively (Fig. 4).

\begin{tabular}{llllll}
\hline Group by WMH Progression & \multicolumn{2}{c}{ Univariate Analysis } & & \multicolumn{2}{c}{ Multivariate Analysis } \\
\cline { 2 - 2 } & OR (95\%CI) & P Value & & OR (95\% CI) & P Value \\
\hline Group 1 $(\leq 0)$ & 1.00 & & & 1.000 & \\
Group 2 $(0,53.64 \%)$ & $3.29(1.51-7.15)$ & 0.003 & & $3.36(1.48-7.67)$ & 0.004 \\
Group 3 $(\geq 53.64 \%)$ & $2.21(1.02-4.81)$ & 0.046 & & $2.14(0.93-4.92)$ & 0.073 \\
\hline
\end{tabular}

$\mathrm{WMH}=$ White matter hyperintensity; OR = Odds Ratio.

\#Odds ratios were adjusted for primary baseline characteristics of patients and the interval between the two MRI scans.

Progression of WMH was associated with occurrence of LI

We next determined the association between WMH progression and LI occurrence using multiple logistic regression analysis. The univariate analysis showed that, compared with Group 1, the risks of LI occurrence were significantly increased in Group 2 (OR, 3.29; 95\% CI, 1.51 to $7.15 ; \mathrm{P}=0.003)$ and Group $3(\mathrm{OR}, 2.21 ; 95 \% \mathrm{CI}$,
1.02 to $4.81 ; \mathrm{P}=0.046$; Table 2). Furthermore, multivariate analysis showed that Group 2 patients still had a higher risk for LI occurrence than Group 1 patients after adjusting for age, sex, vascular history, the interval between the two MRI scans and other baseline confounding factors $(\mathrm{OR}, 3.36,95 \% \mathrm{CI}, 1.48-7.67$, $\mathrm{P}=0.004)$.

Table 3. WMH Progression stratified by time between the two times for MRI.

\begin{tabular}{|c|c|c|c|c|}
\hline \multirow[t]{2}{*}{ Group by WMH Progression } & \multicolumn{2}{|c|}{$\leq 24$ months } & \multicolumn{2}{|c|}{$>24$ months } \\
\hline & OR(95\%CI) & P Value & OR(95\%CI) & P Value \\
\hline Group $1(\leq 0)$ & 1.00 & & 1.000 & \\
\hline Group $2(0,53.64 \%)$ & $3.68(1.51-8.99)$ & 0.004 & $2.40(0.48-11.93)$ & 0.285 \\
\hline Group $3(\geq 53.64 \%)$ & $2.39(0.94-6.05)$ & 0.066 & $1.83(0.40-8.49)$ & 0.438 \\
\hline
\end{tabular}

$\mathrm{WMH}=$ White matter hyperintensity; OR = Odds Ratio.

All odds ratios were adjusted for age, sex, vascular history and other primary baseline confounding factors for LI. 
A

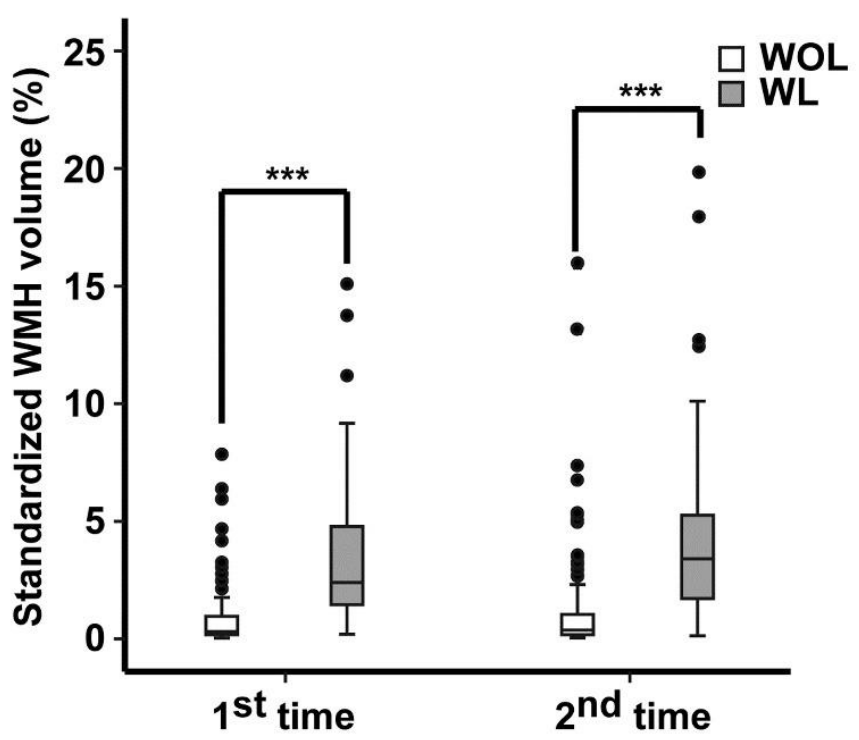

C

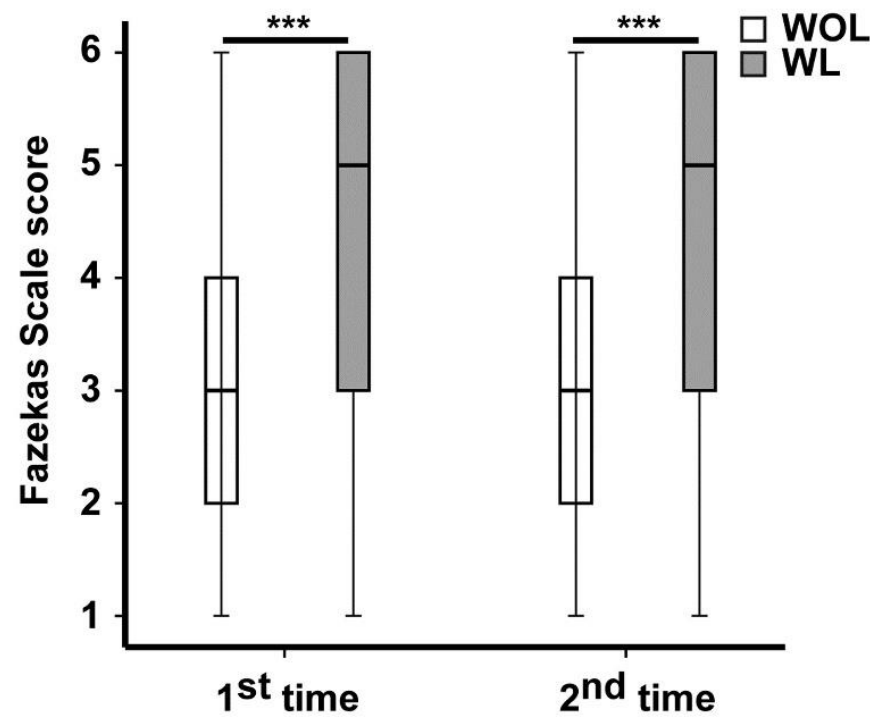

B

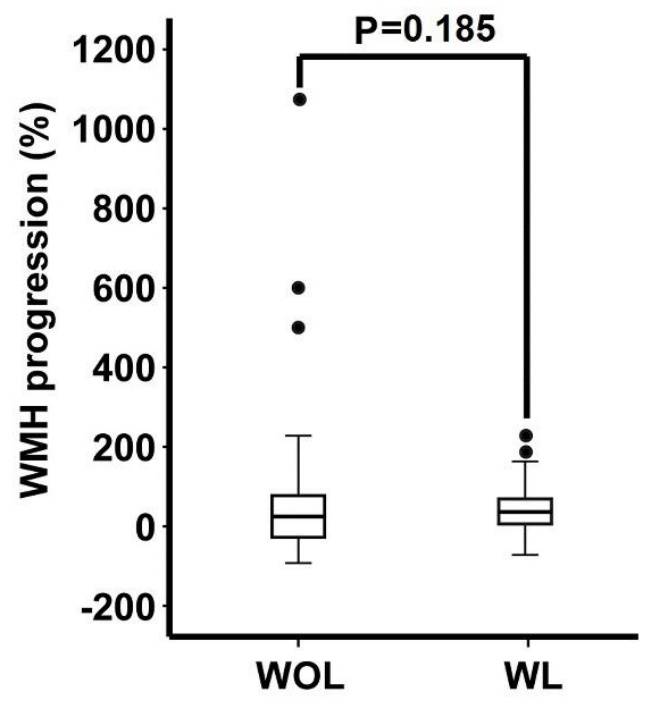

D

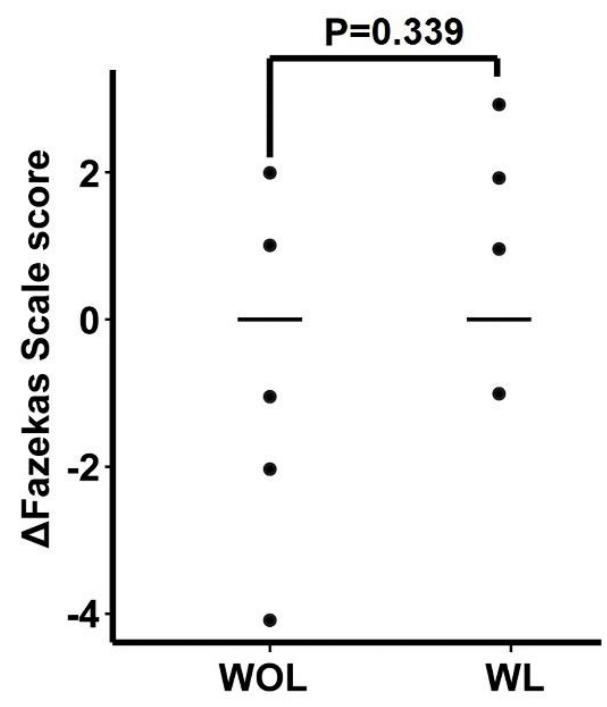

Figure 3. WMH volume and Fazekas scale scores for the $1^{\text {st }}$ and $2^{\text {nd }}$ MRI scans for WL and WOL. (A) Comparison of WMH volumes between two groups for two MRI scans. (B) Comparison of WMH progression between two groups. (C) Comparison of Fazekas scale score between two groups for two MRI scans. (D) Comparison of Fazekas scale score changes between two groups. $\Delta$ Fazekas scale score $=$ the total Fazekas scale score at $2^{\text {nd }}$ MRI scan - the total Fazekas scale score at $1^{\text {st }}$ MRI scan. ${ }^{* * *}$ Significant difference $(\mathrm{P}<0.001)$.

In addition, we assessed whether the associations between WMH progression and LI occurrence were timedependent by dividing patients into two groups: less than or more than 24 months. Multivariate analysis showed that the risk of LI occurrence was greater in Group 2 compared with that in Group 1 within 24 months, after controlling for age, sex and other main baseline confounding factors for LI (OR, 3.68; 95\%CI, 1.51-8.99; $\mathrm{P}=0.004$ ), whereas there was no significant correlation between WMH progression and LI beyond two years
(Table 3).

\section{Discussion}

The main findings of this study were as follows: 1) WMH patients with LI had more severe white matter lesions than patients without $\mathrm{LI}$, according to $\mathrm{WMH}$ quantitative analysis and Fazekas scale score; and 2) WMH progression increased the risk of LI occurrence, particularly within two years. 


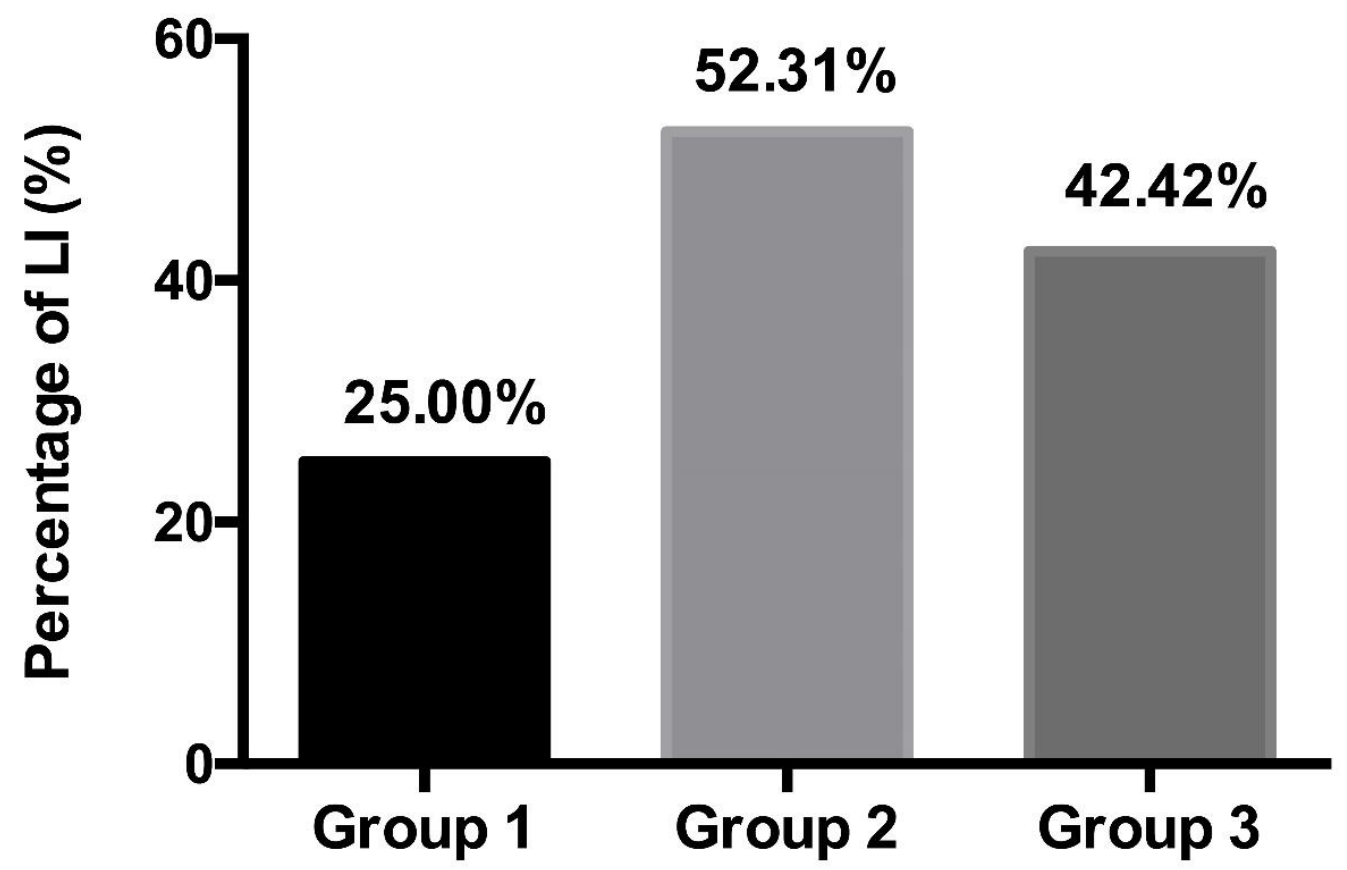

Figure 4. Prevalence of LI in patients stratified by WMH progression.

WMH and LI have certain vascular risk factors in common, such as hypertension, diabetes mellitus, smoking and others [18, 21-23], which was supported by our baseline data. WL subjects had a significantly higher burden of age, SBP, PBG, CRP, diabetes mellitus, and hypertension compared with WOL subjects, whereas TC and ApoA I levels were lower in WL compared with those in WOL patients $(\mathrm{P}<0.05)$. However, when performing multiple logistic regression analysis, we found that these baseline confounding factors did not affect the final conclusions. As for the association between cholesterol levels and LI or WMH, current studies on this topic have conflicting results. Interestingly, one previous study reported that vascular risk factors, such as hypercholesterolemia, hyperlipidaemia and low body mass index seem to have protective effects against WMH progression [24], which is consistent with our results (Table 1).

One previous publication indicated that greater $\mathrm{WMH}$ burden was independently associated with larger volumes of small subcortical infarcts as well as poor 90-day outcomes after a small subcortical infarct [23]. LI frequently coexists with $\mathrm{WMH}$, and both of these processes can lead to cognitive impairment [25]. It was reported that approximately $50 \%$ of LIs were located at the edge of a WMH [26]. Therefore, these studies suggest that WMH progression does increase the risk of LI, although how WMH burden predicts LI occurrence is poorly understood. For the first time, this study shows that
WMH progression increases the risk of LI occurrence. Additionally, we found that the sub-median (0 to 53.64\% WMH progression) rather than the super-median (over $53.64 \% \mathrm{WMH}$ progression) group was associated with the occurrence and development of LI. Or in other words, LIs do not appear to develop when WMH progression has reached a certain load. These results could be interpreted to say that, from a pathological standpoint, decreased vascular density in WMH may decrease the incidence of acute infarctions [26]. On the other hand, it could be that some local LIs overlap with extensive WMH, which would lead to an underestimation of LI incidence.

Furthermore, we found that WMH leading to LI was time dependent and that the risk of LI occurrence was higher within the first 2 years post-WMH. Few longitudinal studies have described the progression of WMH and lacunes. The multicentre, multinational Leukoaraiosis and Disability study included a total of 396 patients who were followed for 3 years, and results showed that one in five subjects showed at least one new lacune within 3 years, and the new lacunes in a relatively high proportion of subjects were associated with stratification by WMH severity [18]. Moreover, Schmidt reported that of 273 participants (mean age 60 years), 17.9\% individuals had WMH progression and $2.2 \%$ had a new LI within 3 years [27]. Therefore, it appears that early treatment of WMH progression would be beneficial.

Some studies have indicated that WMH is secondary to LI [22], whereas others have reported that WMH 
promotes the development of LI [28]. One potential mechanism of the former is that lacunar infarcts affect white matter tract integrity [22]. By contrast WMHs might promote LI by lowering cerebral blood flow (CBF) in the WMH [28]. Traylor M. and colleagues found that WMHassociated genetic variants also increased the risk of lacunar infarcts, and therefore, WMH and LI may share pathogeneses [29].

\section{Limitations}

This study has several limitations. First, this was a singlecentre, retrospective cohort research plan, which should not be extensively relied on in clinical practice. Second, although a large number of patients over six years of records were screened, only 187 patients met the inclusion criterion, which lowers the statistical power of this study. A future study involving multiple centres and more patients would help to determine the accuracy of these findings.

\section{Conclusions}

We show that WMH progression was significantly associated with the occurrence of LI within a certain time frame and that WMH progression may serve as an independent indicator of LI occurrence. Our study suggests that WMH should be addressed at the early stages of evolution in clinical practice.

\section{Acknowledgements}

This work was supported by the National Key Research and Development Program of China (2016YFC1300500$504)$, the National Natural Science Foundation of China (81230026, 81630028), the Natural Science Foundation (BE2016610) of Jiangsu Province, the Jiangsu Provincial Key Medical Discipline (ZDXKA2016020) and National Health and Family Planning Commission in China (GN2016R0005).

\section{References}

[1] Wardlaw JM, Smith C, Dichgans M (2013). Mechanisms of sporadic cerebral small vessel disease: insights from neuroimaging. Lancet Neurol, 12: 483497

[2] Choi JC (2015). Genetics of cerebral small vessel disease. J Stroke, 17: 7-16

[3] Hinman JD, Lee MD, Tung S, Vinters HV, Carmichael ST (2015). Molecular disorganization of axons adjacent to human lacunar infarcts. Brain, 138: 736-745

[4] Pinter D, Enzinger C, Fazekas F (2015). Cerebral small vessel disease, cognitive reserve and cognitive dysfunction. J Neurol, 262: 2411-2419
[5] Wardlaw JM, Smith EE, Biessels GJ, Cordonnier C, Fazekas F, Frayne R, et al. (2013). Neuroimaging standards for research into small vessel disease and its contribution to ageing and neurodegeneration. Lancet Neurol, 12: 822-838

[6] Moran C, Phan TG, Srikanth VK (2012). Cerebral small vessel disease: a review of clinical, radiological, and histopathological phenotypes. Int J Stroke, 7: 3646

[7] Pantoni L (2010). Cerebral small vessel disease: from pathogenesis and clinical characteristics to therapeutic challenges. Lancet Neurol, 9: 689-701

[8] Wu YF, Wu WB, Liu QP, He WW, Ding H, Nedelska Z, et al. (2016). Presence of lacunar infarctions is associated with the spatial navigation impairment in patients with mild cognitive impairment: a DTI study. Oncotarget, 7: 78310-78319

[9] Leeuw FD, Groot JCD, Achten E, Oudkerk M, Ramos LMP, Heijboer R, et al. (2001). Prevalence of cerebral white matter lesions in elderly people: a population based magnetic resonance imaging study. The Rotterdam Scan Study. J Neurol Neurosurg Psychiatry, 70: 9-14

[10] Smith EE, Saposnik G, Biessels GJ, Doubal FN, Fornage M, Gorelick PB, et al. (2017). Prevention of Stroke in Patients With Silent Cerebrovascular Disease: A Scientific Statement for Healthcare Professionals From the American Heart Association/American Stroke Association. Stroke, 48: e44-e71

[11] Wen W, Sachdev PS (2004). Extent and distribution of white matter hyperintensities in stroke patients: the Sydney Stroke Study. Stroke, 35: 2813-2819

[12] Fanning JP, Wong AA, Fraser JF (2014). The epidemiology of silent brain infarction: a systematic review of population-based cohorts. BMC Med, 12: 119

[13] Allan CL, Zsoldos E, Filippini N, Sexton CE, Topiwala A, Valkanova V, et al. (2015). Lifetime hypertension as a predictor of brain structure in older adults: cohort study with a 28-year follow up. Br J Psychiatry, 206: 308-315

[14] Srikanth V, Phan TG, Chen J, Beare R, Stapleton JM, Reutens DC (2010). The location of white matter lesions and gait--A voxel-based study. Ann Neurol, 67: 265-269

[15] Callisaya ML, Beare R, Phan T, Blizzard L, Thrift AG, Chen J, et al. (2015). Progression of white matter hyperintensities of presumed vascular origin increases the risk of falls in older people. J Gerontol A Biol Sci Med Sci, 70: 360-366

[16] Edwards JD, Jacova C, Sepehry AA, Pratt B, Benavente OR (2013). A quantitative systematic review of domain-specific cognitive impairment in lacunar stroke. Neurology, 80: 315-322

[17] Goldberg I, Auriel E, Russell D, Korczyn AD (2012). Microembolism, silent brain infarcts and dementia. J Neurol Sci, 322: 250-253

[18] Gouw AA, van der Flier WM, Fazekas F, van Straaten EC, Pantoni L, Poggesi A, et al. (2008). Progression of white matter hyperintensities and incidence of new 
lacunes over a 3-year period: the Leukoaraiosis and Disability study. Stroke, 39: 1414-1420

[19] Fazekas F, Chawluk JB, Alavi A, Hurtig HI, Zimmerman RA (1987). MR signal abnormalities at 1.5 $\mathrm{T}$ in Alzheimer's dementia and normal aging. AJR Am J Roentgenol, 149: 351-356

[20] Boutet C, Rouffiange-Leclair L, Schneider F, Camdessanche JP, Antoine JC, Barral FG (2016). Visual Assessment of Age-Related White Matter Hyperintensities Using FLAIR Images at $3 \mathrm{~T}$ : Inter- and Intra-Rater Agreement. Neurodegener Dis, 16: 279-283

[21] Luo Y, Li J, Zhang J, Xu Y (2014). Low HDL cholesterol is correlated to the acute ischemic stroke with diabetes mellitus. Lipids Health Dis, 13: 171

[22] Imaizumi T, Inamura S, Nomura T, Kanno A, Kim SN (2015). The Severity of White Matter Lesions Possibly Influences Stroke Recurrence in Patients with Histories of Lacunar Infarctions. J Stroke Cerebrovasc Dis, 24: 2154-2160

[23] Helenius J, Mayasi Y, Henninger N (2017). White matter hyperintensity lesion burden is associated with the infarct volume and 90-day outcome in small subcortical infarcts. Acta Neurol Scand, 135: 585-592
Mok V, Kim JS (2015). Prevention and Management of Cerebral Small Vessel Disease. J Stroke, 17: 111-122

Yan T, Yu JR, Zhang YP, Li T (2015). Analysis on correlation of white matter lesion and lacunar infarction with vascular cognitive impairment. Int J Clin Exp Med, 8: $14119-14122$

[26] Zhang X, Ding L, Yuan J, Qin W, Hu W (2015). Spatial Relationship between Acute Lacunar Infarction and White Matter Hyperintensities. Eur Neurol, 74: 259266

[27] Schmidt R, Fazekas F, Enzinger C, Ropele S, Kapeller P, Schmidt H (2002). Risk factors and progression of small vessel disease-related cerebral abnormalities. J Neural Transm Suppl, 62: 47-52

[28] Reijmer YD, Freeze WM, Leemans A, Biessels GJ, Utrecht Vascular Cognitive Impairment Study G (2013). The effect of lacunar infarcts on white matter tract integrity. Stroke, 44: 2019-2021

[29] Traylor M, Rutten-Jacobs LC, Thijs V, Holliday EG, Levi C, Bevan S, et al. (2016). Genetic Associations With White Matter Hyperintensities Confer Risk of Lacunar Stroke. Stroke, 47: 1174-1179 